\title{
Neue veränderliche Sterne
}

mitgetheilt von $A$. W. Roberts in A. J. 508.

Var. 91.1901 Velorum $10^{\mathrm{h}} \mathrm{r}^{\mathrm{m}} 44^{\mathrm{s}}-41^{\circ} 43^{\prime} 8$ (1875) Algoltyp. Gr. 10.0-10.9. Periode $1^{\mathrm{d}} 20^{\mathrm{h}} 30^{\mathrm{m}} 2^{\mathrm{s}}$.

Var. 92 . 1901 Coronae austr. ${ }^{1} 8^{\mathrm{h}} 3^{2 \mathrm{~m}} 45^{\mathrm{s}}-37^{\circ} 35^{\prime} 8$ (1875) wahrscheinlich veränderlich. Gr. 8.0-9.0. Periode wahrscheinlich 185 Tage.

\section{Neuer Veränderlicher 93.1901 Sagittae vom Algoltypus. BD. $+19^{\circ} 397519^{\mathrm{h}} 14^{\mathrm{m}} 26^{\mathrm{s}}+19^{\circ} 25^{\prime} 4$ (1900).}

Bei Beobachtungen des Sterns

Pots. Durchm. Zone $0^{\circ}-20^{\circ}$ No. $2729=$ BD. + I $9^{\circ} 3975$

habe ich denselben als Veränderlichen vom Algoltypus erkannt. Während die dauernde Helligkeit etwa $=6{ }^{\mathrm{m}} \cdot 5$ ist, sinkt sie im kleinsten Licht bis zur 9. Gr. herab, bleibt hier einige Zeit ziemlich unverändert, worauf wieder raschere Zunahme erfolgt. Da es bisher noch nicht gelang, den Gesammtverlauf des Lichtwechsels zu verfolgen, sowie die Periodenlänge zu ermitteln, so sollen die folgenden vorIlmenau, I 901 Nov. 5. läufigen Angaben nur als Anhalt fừr weitere Beobachtungen dienen.

Die Lichtcurve scheint grosse Aehnlichkeit mit der von $U$ Cephei aufzuweisen, während die Periode etwa $I 7^{d}$, wahrscheinlich wohl nur $1 / 2$ oder $1 / 3$, selbst $1 / 5$ dieser Zeit sein wird, da andere Combinationen nach den vorliegenden Vergleichen kaum zulässig erscheinen. Das letzte, unvollständig beobachtete kleinste Licht fiel auf Nov. I $61 \%^{\mathrm{h}}$ M. Z. Gr., so dass am 18 . November und 5 . December weitere Minima zu erwarten sein dürften.

\section{F. Schwab.}

\section{Mittheilungen über kleine Planeten.}

(291) Alice. Die diesem Planeten in Nr. 3746 p. 29 zugeschriebenen Beobachtungen sind zu streichen. Der Planet ist nachträglich auf der Platte 1901 Oct. $129^{\mathrm{h}} 18^{\mathrm{m}} \cdot 6 \mathrm{M}$. Z. Heid. in der Position $23^{\mathrm{h}} 27^{\mathrm{m}} \cdot 3-4^{\circ} 44^{\prime}(1855.0)$ aufgefunden worden: Wolf.

(346) Hermentaria. Corr. der Ephemeride (Veröff. R. I. I 5): Oct. $3^{1}-2^{\mathrm{m}} 4^{8^{\mathrm{s}}}-\mathbf{1} 7^{\prime} 6$. Gr. 9:8. W. Luther.

(380) Fiducia. $190 \mathrm{r}$ Nov. $48^{\mathrm{h}} 57^{\mathrm{m}} \cdot 4$ M. Z. Heid. $2^{\mathrm{h}} 7^{\mathrm{m}} \cdot 5+3^{\circ} 33^{\prime}$. Wolf und Kopff.

(384) Burdigala. Corr. der Ephemeride (Veröff. R. I. I5): Nov. $3-2^{\mathrm{m}} \mathrm{o}^{\mathrm{s}}-\mathbf{2}$.'o. Gr. $10^{\mathrm{m}} \cdot \mathbf{9}$. W. Luther.

(423) Diotima und r90 I GV. Nach Mittheilungen von Herrn $A$. Berberich und Prof. $M$. Wolf gehört die dem Planeten (423) in Nr. 3746 p. 30 zugeschriebene Beobachtung nicht diesem, sondern dem Planeten I90I GV an. Die Correction der Ephemeride von (423) in Veröff. R. I. Nr. I 5 beträgt nach einer Beobachtung von Dr. Villiger: Oct. I r $-5^{\mathrm{m}} \circ-43^{\prime}$. Der Planet $1901 \mathrm{GV}$ ist auch Oct. I 8 von Miss Mary $W$. Whitney auf dem Vassar College Observatory aufgefunden und dort gleichfalls zunächst für (423) gehalten worden.

(434) Hungaria 1901 Nov. $4 \mathrm{I}^{\mathrm{h}} 5^{\mathrm{m}} \cdot 4^{4}$ M. Z. Heid. $4^{\mathrm{h}} \mathrm{I}^{\mathrm{m}} \cdot 3-\mathrm{I}^{\circ} 24^{\mathrm{\prime}}$. Wolf.

I $901 \mathrm{GZ}$. $190 \mathrm{I}$ Nov. $27^{\mathrm{h}} 33^{\mathrm{m}} \cdot 4$ M. Z. Heid. $1^{\mathrm{h}} 3^{\mathrm{m}} \cdot{ }^{\mathrm{m}}+17^{\circ} \mathrm{g}^{\circ}$. Carnera.

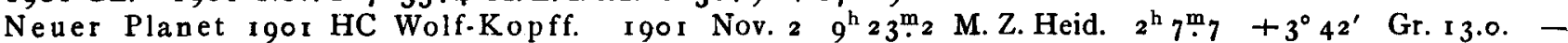
Nov. $48^{\mathrm{h}} 57^{\mathrm{m}} \cdot 4$ M. Z. Heid. $2^{\mathrm{h}} 6^{\mathrm{m}} \cdot 3+3^{\circ} 27^{\prime}$ Wolf.

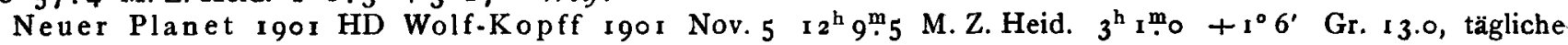
Bewegung $-\mathbf{I} \mathbf{m}_{\mathbf{I}}+\mathbf{I}^{\prime}$. Wolf.

Neuer Planet 190r HE Kopff I $90 \mathrm{I}$ Nov. $31^{\mathrm{h}} \mathrm{I}^{\mathrm{m}} \cdot 4$ M. Z. Heid. $2^{\mathrm{h}} \mathbf{2}^{\mathrm{m}} \cdot 6+8^{\circ} 34^{\prime}$ Gr. I3.5, tägliche Bewegung $-0 .{ }^{\mathrm{m}} 8 \mathrm{-}-5^{\prime}$. Wolf.

Neuer Planet I90I HF Wolf I90I Nov. 6 I $2^{\mathrm{h}} 56^{\mathrm{m}} \cdot 5$ M. Z. Heid. $4^{\mathrm{h}} 59^{\mathrm{m}} \cdot 6+13^{\circ}$ I $7^{\prime}$ Gr. II.5, tägliche Bewegung $-0^{\mathrm{m}} \cdot \mathrm{q} \mathrm{o}^{\prime}$. Wolf.

Neuer Planet rgor HG Wolf.Carnera rgor Nov. 6 1 $2^{\mathrm{h}} 56^{\mathrm{m}_{5}} \cdot \mathrm{M}$. Z. Heid. $5^{\mathrm{h}} 8^{\mathrm{m}} \cdot 3+18^{\circ} 14^{\prime}$ Gr. 1 , tägliche Bewegung $-0 .{ }^{\mathrm{m}} 6+3^{\prime}$. Wolf.

Nova (3.1901) Persoi. Telegramm aus Cambridge, Mass., d. d. I1. November: »From Crossley photographs. Perrine finds that four principal condensations faint nebula surrounding Nova Persei moved south east, one minute arc in six weeks. Pickering. \&

Personalnotiz. Die Adresse von Dr. Dorothea Klumpke, bisher Sternwarte Paris, ist nach ihrer Verheirathung mit Dr. Roberts, Mrs. Isaac Roberts, Starfield, Crowborough, Sussex, England.

Inhalt zu Nr. 3747-48. C. F. Merfield. Definitive Orbit Elements of Comet I 899 I. 33. - Ö. Bergstrand. Ueber die Eigenbewegung der Nova (3.1901) Persei. 77. - A.W. Roberts. Neue veränderliche Sterne. 79. $-F$. Schwab. Neuer Veränderlicher 93.1901 Sagittae vom Algoltypus. 79. - Mittheilungen über kleine Planeten. 79. - Nova (3. I 9or) Persei. 79. - Personalnotiz. 79. 